\title{
Aneurysms of the Sinus of Valsalva Associated with Severe Aortic Valve Regurgitation
}

\author{
Hao Thai Phan \\ Department of Internal Medicine, Pham Ngoc Thach University of Medicine, Ho Chi Minh City, Vietnam.
}

\author{
Corresponding Author: \\ Dr. Hao Thai Phan \\ Email:phanthaihao@yahoo.com \\ This is an Open Access article distributed \\ under the terms of the Creative Commons \\ Attribution License (creativecommons.org/ \\ licenses/by/3.0). \\ Published

\begin{abstract}
Background: Sinus of Valsalva aneurysms (SOVA) are very uncommon, with an incidence ranging from 0.1 to $3.5 \%$ of all congenital heart defects. Such aneurysms account for only $0.14 \%$ of all open heart surgical procedures. SOVA are usually diagnosed as an incidental finding or after an acute rupture into an adjacent cardiac structure. Case Report: We report a case of SOVA associated with severe aortic valve regurgitation. Conclusion: Before rupture, SOVA may present with conduction-system abnormalities attributable to erosion into the inter-ventricular septum, thromboembolism originating in the aneurysm sac, and myocardial ischemia attributable to coronary compression.
\end{abstract}

Keywords: Aortic Valve, Aortic Valve Insufficiency, Congenital Heart Defects, Coronary Artery Disease, Sinus of Valsalva.

\section{Introduction}

Sinus of Valsalva aneurysm (SOVA) is a rare clinical entity. Clinical manifestations can vary from an incidental finding on an imaging study to a life-threatening emergency. We report a case of SOVA associated with severe aortic valve regurgitation. Surgical repair of the aneurysm with reimplantation of the right and left coronary arteries was performed in addition to aortic valve replacement (Bentall procedure). The patient had an uneventful post-operative course and remains asymptomatic at the three-month follow-up visit.

\section{Case Report}

A 35-year-old female with no past significant medical history was admitted for exertional dyspnea during last two weeks. There was no relevant family history. Her cardiovascular physical examination was significant for IV/VI early diastolic murmur at third left inter-costal space and other peripheral signs: Corrigan pulse,
Hill's sign, Duroziez's murmur. Her admission ECG [Fig.1] showed sinus rhythm with a heart rate of $91 \mathrm{bpm}$, left ventricular hypertrophy. Her portable chest X-ray [Fig.2] was significant for cardiomegaly. Her transthoracic echocardiogram revealed a massively enlarged aortic root with large sinuses of Valsalva aneurysms measuring $\approx 7.5 \mathrm{~cm}$ in diameter [Fig.3]. Severe aortic regurgitation was present, and left ventricular function was normal. The patient was further evaluated by computerized tomography of the ascending aorta [Fig.4a-d]. This imaging modality further confirmed the findings in the echocardiogram. These findings were not previously known by the patient. The patient denied any signs or symptoms of cardiac ischemia or embolic complications such as stroke. Subsequently, the patient underwent replacement of the aortic valve with mechanical prosthesis and Dacron conduit, with reimplantation of the right and left coronary arteries on the anterior and lateral surfaces, respectively (Bentall procedure). The post-operative course was uneventful. She remains 
asymptomatic at the three-month follow-up visit. Repeat echocardiography shows no residual abnormalities.

\section{Discussion}

SOVA is a rare but serious cardiac anomaly. It was first described by Hope in 1839 [1]. Incidence varies from $0.1-3.5 \%$ among all congenital cardiac lesions [2]. The left SOVA is the rarest of all SOVAs $(<5 \%)[3,4]$. SOVA can be congenital or acquired. The right coronary sinus is by far the most common site of origin for congenital SOVAs. These are usually associated with other congenital cardiac defects. Ventricular septal defects and aortic regurgitation are the most common co-existing cardiac defects. Acquired aneurysms are mostly caused by trauma, atherosclerosis, endocarditis, syphilis, and connective tissue diseases [2].

Most SOVAs result from a congenital deficiency of elastic lamellae in the wall of the affected sinus, with separation of the media in the sinus of Valsalva from the media adjacent to the aortic valve annulus [5]. Eventually, this weak area enlarges and becomes aneurysmal due to the effect of high aortic pressure. Clinical manifestations may vary from an incidental finding on an imaging study to a life-threatening emergency.

Unruptured SOVAs typically remain asymptomatic for decades and are often discovered incidentally on echocardiography or other imaging modalities. However, large unruptured aneurysms can produce mass effect and may cause valvular dysfunction, right ventricular outflow tract obstruction, and myocardial ischemia due to compression on the origin of the main coronary arteries [6]. Nonetheless, acute symptoms usually occur in most cases of aneurysmal rupture and subsequent fistula formation to the adjacent cardiac chamber. Acute symptoms consist of sudden shortness of breath and chest pain. Moreover, a characteristic loud continuous murmur accompanied with a thrill is often related to rupture of the aneurysm [7].

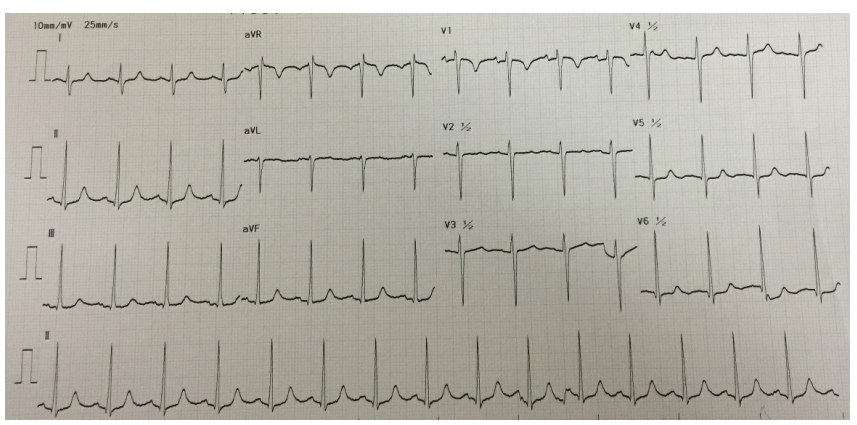

Fig.1: ECG showing sinus rhythm and left ventricular hypertrophy.

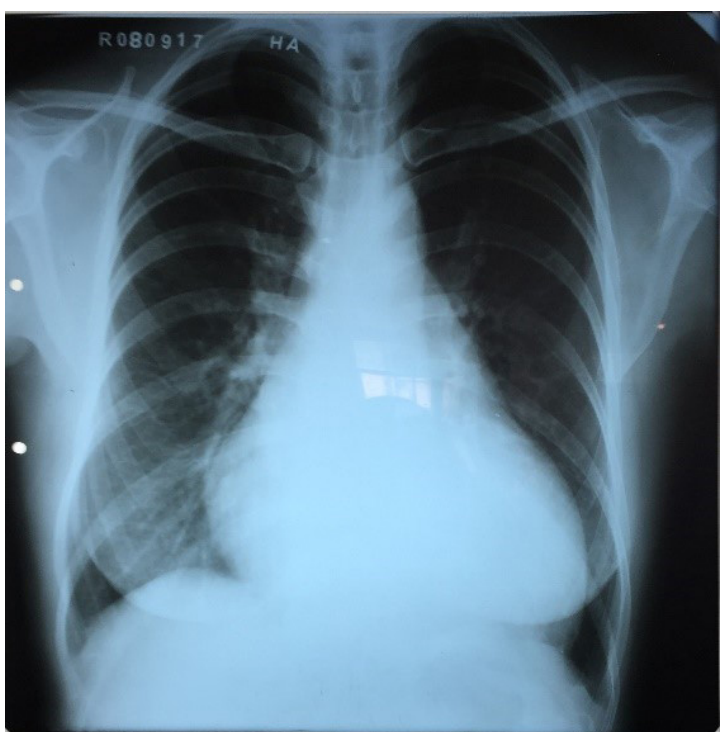

Fig.2: Cardiomegaly seen on chest X-ray.

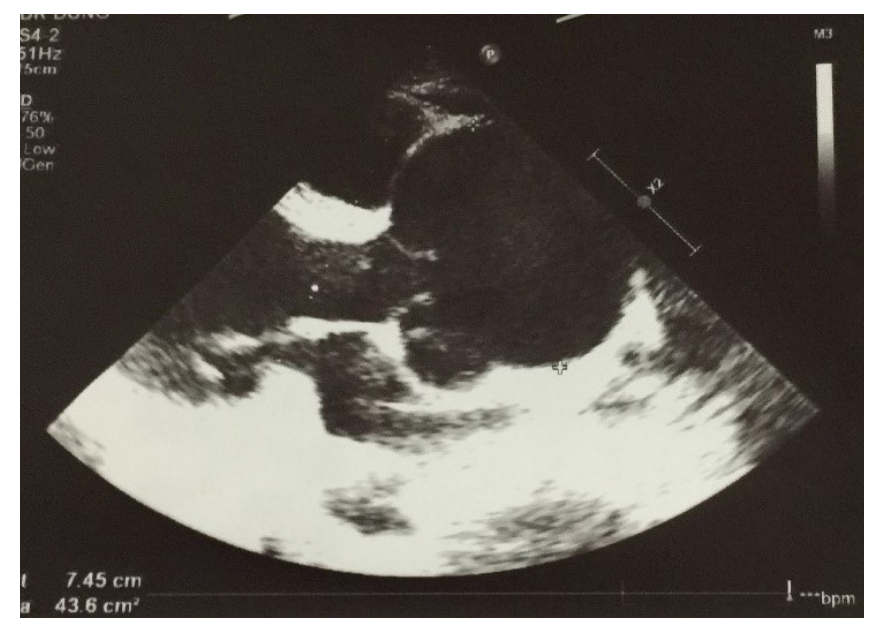

Fig.3: Echocardiography suggestive of sinus of Valsalva aneurysms. 
Congenital SOVAs usually rupture during the third and fourth decade of life. Generally, aneurysms rupture into the right ventricle followed by right atrium. In contrast, left aneurysms rupture into the pericardial cavity. Other potential complications of SOVAs include aortic regurgitation, myocardial ischemia, conduction abnormalities, and thrombus formation with subsequent systemic embolization [8]. Nonetheless, heart failure is still the main cause of death in patients with SOVAs. Echocardiography is very useful in the diagnosis of SOVAs and associated lesions such as aortic regurgitation, ventricular septal defects, and endocarditis [9]. Other diagnostic modalities such as CTA, cardiac MRI, and cardiac catheterization with aortography are often necessary to confirm diagnosis, identify coronary anatomy, and detect complications. Surgical repair is the treatment of choice for ruptured SOVAs with excellent long term survival of $97 \%$ at 10 years $[10,11]$. An unruptured but symptomatic SOVA should also be considered for surgical intervention. Moreover, concomitant ventricular septal defects, endocarditis, and moderate to severe aortic valve regurgitation require surgery [12]. Conversely, several reports have shown that percutaneous closure of ruptured SOVA using transcatheter closure devices is feasible and effective therapeutic alternative to surgery [13-15].

\section{Conclusion}

Aneurysms of sinus of Valsalva are rare congenital or acquired cardiac defects that have been increasingly diagnosed as a result of improved imaging techniques. This is a case of aneurysms of all three coronary sinuses presenting with no clinical signs or symptoms. Despite being asymptomatic and having a favorable course thus far, we recommended that our patient have surgery performed as soon as possible to minimize the risk of future cardiovascular events.

Contributors: HTP wrote the manuscript, did literature search, involved in patient management, and approved the final version of this article. He will act as guarantor of the study.

Funding: None; Competing interests: None stated.
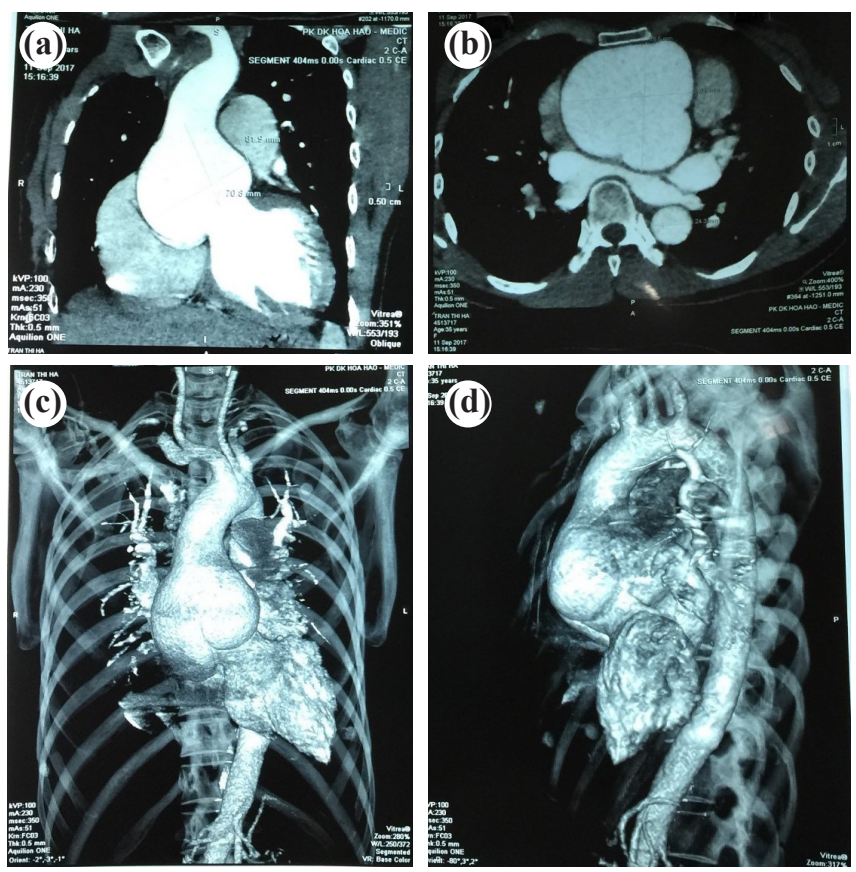

Fig.4(a-d): Chest MS CT show large sinuses of Valsalva aneurysms.

\section{References}

1. Hope J. A Treatise on the diseases of the heart and great vessels, John Churchill, London, UK, $3^{\text {rd }}$ edition, 1839.

2. Goldberg N, Krasnow N. Sinus of valsalva aneurysms. Clinical Cardiology. 1990;13:831-836.

3. Moustafa S, Mookadam F, Cooper L, Adam G, Zehr KJ, Stulak J, et al. Sinus of valsalva aneurysms 47 years of a single center experience and systematic overview of published reports. American Journal of Cardiology. 2007;99:1159-1164.

4. Smer A, Elsallabi O, Ayan M. Sinus of Valsalva aneurysm: A rare cause of dyspnea. Case Reports in Medicine. 2015;467935.

5. Edwards JE, Burchell HB. Specimen exhibiting the essential lesion in aneurysm of the aortic sinus. Proceedings of the Staff Meetings of the Mayo Clinic. 1956;31:407-412.

6. Martínez-Comendador J, Gualis J, Martín CE, Santamaria A. Left main coronary artery compression by a left sinus of Valsalva aneurysm. Interactive Cardiovascular and Thoracic Surgery. 2013;16:713-714.

7. Morch JE, Greenwood WF. Rupture of the sinus of valsalva. A study of eight cases with discussion on the differential diagnosis of continuous murmurs. The American Journal of Cardiology. 1966;18:827-836.

8. Stöllberger C, Seitelberger R, Fenninger C, Prainer C, Slany J. Aneurysm of the left sinus of Valsalva. An unusual source of cerebral embolism. Stroke. 1996;27:1424-1426. 
9. Cheng TO, Yang YL, Xie MX, Wang XF, Dong NG, $\mathrm{Su} \mathrm{W}$, et al. Echocardiographic diagnosis of sinus of Valsalva aneurysm: a 17-year (1995-2012) experience of 212 surgically treated patients from one single medical center in China. International Journal of Cardiology. 2014;173:33-39.

10. Au WK, Chiu SW, Mok CK, Lee WT, Cheung D, He GW. Repair of ruptured sinus of Valsalva aneurysm: determinants of long-term survival. Annals of Thoracic Surgery. 1998;66:1604-1610.

11. Sarikaya S, T. Adademir T, Elibol A, Büyükbayrak F, Onk A, Kirali K. Surgery for ruptured sinus of Valsalva aneurysm: 25-year experience with 55 patients. European Journal of Cardio-Thoracic Surgery, 2013;43:591-596.

12. Yan F, Abudureheman M, Huo Q, Shabiti A, Zhu T, Liu
Z. Surgery for sinus of Valsalva aneurysm: 33-year of a single center experience. Chinese Medical Journal. 2014;127:4066-4070.

13. Arora R, Trehan V, Rangasetty UMC, Mukhopadhyay S, Thakur AK, Kalra GS. Transcatheter closure of ruptured sinus of valsalva aneurysm. Journal of Interventional Cardiology. 2004; 17:53-58.

14. Kuriakose EM, Bhatla P, McElhinney DB. Comparison of reported outcomes with percutaneous versus surgical closure of ruptured sinus of valsalva aneurysm. The American Journal of Cardiology. 2015;115:392-398.

15. Sinha SC, Sujatha V, Mahapatro AK. Percutaneous transcatheter closure of ruptured sinus of Valsalva aneurysm: immediate result and long-term follow-up. International Journal of Angiology. 2015;24:99-104. 